\title{
El cine como referente de los grupos musicales españoles en la década de 1980
}

\author{
Juan Carlos Rodríguez CENTENO \\ Universidad de Sevilla \\ jcrodri@us.es
}

\begin{abstract}
Resumen:
Uno de los períodos musicales más brillantes de la historia de la música popular española se produjo en la década de 1980. Una nueva generación surgida en la Transición, influenciada por los movimientos culturales en general, y musicales en particular, que se estaban produciendo en Estados Unidos y Reino Unido, propició la aparición de numerosas formaciones musicales que tuvieron en el cine un punto de referencia y una fuente de inspiración. Este trabajo se centra en analizar dichas influencias.
\end{abstract}

Palabras clave: Música pop y rock; cine.

The cinema as model for Spanish popular music in the $80^{\prime} \mathrm{s}$.

\begin{abstract}
:
One of the most outstanding periods in Spanish popular music occurred in the eighties. A younger generation emerged from the Spanish transition to democracy, influenced by other cultural movements in general, especially in music, which were taking place in the USA and the UK. This led to the birth of a countless number of musical bands, for which the cinema brought a significant point of reference and a never-ending source of inspiration. The aim of this research is to analyse these influences.
\end{abstract}

Key Words: Pop and rock music; cinema.

\section{Referencia normalizada:}

Rodríguez Centeno, J. C. (2014): El cine como referente de los grupos musicales españoles en la década de 1980. Historia y Comunicación Social. Vol. 19. Núm. Especial Febrero. Págs. 667-676.

Sumario: 1. Introducción. 2. Metodología. 3. Los nombres. 4. Las portadas. 5. Los discos. 6. Los géneros. 7. Los grupos españoles en el cine. 8. Panorámica por la historia del cine. 9. Conclusión. 10. Bibliografía.

\section{Introducción.}

A finales de los años setenta España era un país en transformación. Había finalizado una larga dictadura y los españoles se abrían entre ilusionados y temerosos a un nuevo futuro. De ese magma social emerge una nueva generación nacida en los estertores del Franquismo, deseosos de salir del marasmo cultural de un país adocenado, y que encuentra en el movimiento punk y sus inmediatos postpunk y new wave proce- 
dentes de Estados Unidos y Reino Unido una nueva identidad. Con Madrid como epicentro, en los inicios de su posteriormente tan manida y afamada Movida, pero con una intensa actividad en otras muchas ciudades como Barcelona, Vigo, Valencia, Granada entre otras, surgirán por todo el país multitud de formaciones musicales que convertirían la década de los ochenta en una de las épocas más fértiles de la historia de la cultura popular española.

La temática desarrollada en la discografía de las formaciones de aquellos años es, en buena lógica, múltiple y variada, y en consecuencia no es el objetivo de este estudio su catalogación y análisis. Nos centramos en la influencia del cine en los grupos que surgieron a finales de los setenta y principios de los ochenta y que tuvieron discografía editada.

\section{Metodología.}

Aunque algunas formaciones de las aquí incluidas iniciaron su actividad musical a finales de los setenta, y otras continuaron su carrera en décadas posteriores, e incluso alguna siguen activas, o se dieron casos de bandas separadas que al cabo de los años vuelven a reunirse y editar discos y hacer giras, el requisito para ser incluidas en este estudio es que el grueso de su carrera discográfica transcurriera en la década de los ochenta, o que sus mayores éxitos se produjeran en dichas fechas. Hemos utilizado como fuente principal la web lafonoteca.net que tiene catalogados a trescientos doce grupos españoles en la década seleccionada. Hemos estudiado la discografía de cada uno de los grupos buscando las referencias cinematográficas en los nombres de las formaciones, las canciones y las portadas de los discos. Sin embargo no todos los grupos que triunfaron en los ochenta aparecen en dicha web por lo que tuvimos que buscar fuentes alternativas, como la bibliografía abajo mencionada, en especial las publicaciones de Jesús Ordovás, y las páginas web personales de las formaciones. También hemos recurrido en busca de datos y cifras sobre espectadores, películas, recaudaciones y protagonistas a la web mundocine.net.

\section{Los nombres.}

Desde los inicios de la Nueva Ola madrileña se observa que el cine es una referencia común y recurrente entre las nuevas formaciones, y el primer indicador lo encontramos en los nombres de los grupos. Así, de las cenizas del seminal Kaka de Luxe, surge Paraíso, a cuyo frente está uno de los músicos más prolíficos y activos del nuevo movimiento, Fernando Márquez "El Zurdo", que se inspira en el musical de terror de Brian de Palma El fantasma del paraíso (Phantom of the Paradise, 1974), para dar nombre a su efímero grupo. 
A principios de la década de los ochenta tres estudiantes universitarios seguidores del afterpunk británico forman Gabinete Caligari, un título que toman prestado de la obra cumbre del Expresionismo cinematográfico alemán El gabinete del doctor Caligari (Das Cabinet des Dr. Caligari, 1920), un film y un movimiento cultural que comparten universo imaginario y temático con el rock siniestro: personajes maléficos y desequilibrados; culto a la muerte, al crimen y al dolor; y estética oscura y tenebrosa.

Insertos en esta misma corriente siniestra, aunque menos radicalizados, encontramos a Décima Víctima, una formación liderada por un "veterano" de la escena musical madrileña, Carlos Entrena (Kaka de Luxe, Paraíso, Ejecutivos Agresivos), que se inspira en el filme futurista italiano La decima vittima (1965), protagonizado por Macello Mastroianni y Ursula Andress.

Parece evidente que los grupos adscritos a la corriente del afterpunk o rock siniestro tenían como una de sus habituales referencias el universo cinematográfico, y entre ellos encontramos también a la formación Polanski y el Ardor, que evidentemente tomaron prestado su nombre del realizador polaco más famoso y controvertido.

Como en un caso anterior, el cine futurista sirve de referencia para nominar al grupo Alphaville, aunque en esta ocasión se trata de una película francesa, la mítica obra que Jean-Luc Godard dirigió en 1965.

Un film de gángsters interpretado por el gran icono del género en sus primeros años, James Cagney, sirvió de inspiración a los Hombres G (G-Men, 1935), probablemente la formación musical más cinéfila de la historia del pop español, como veremos a lo largo de este artículo a tenor de las numerosas referencias en su discografía. La causa de la cinefilia se debe a que el cantante y líder de la formación, David Summers, es hijo del cineasta Manuel Summers, que dirigió las dos películas que Hombres $\mathrm{G}$ rodaron en la cúspide de su carrera.

Otro de los grupos en los que encontramos numerosas referencias en torno al cine son Objetivo Birmania, formación deudora en su nombre del clásico del cine bélico rodado por Raoul Walsh (Objetive Burma, 1945), al frente de cuyo extenso reparto se encontraba la estrella de Hollywood Errol Flynn.

Los amantes de María fueron una formación barcelonesa de escasa trayectoria, en la que solo publicaron un disco, que tomaron su nombre de la película de Andrei Konchalovski (Maria's lovers, 1984), protagonizada por Natassja Kinski, Robert Mitchum y Keith Carradine. Este último pertenece a una de las sagas más célebres del cine y la televisión, iniciada por el patriarca John Carradine (La diligencia, Las uvas de la ira), y seguida por los hermanos David, que adquirió fama mundial al protagonizar la serie televisiva Kung Fu; Keith (Los duelistas); y el menos conocido Robert (The long ryders). En honor a esta saga adoptaron su nombre los barceloneses Los Carradine.

A mediados de la década de los ochenta surgió en Zaragoza una formación de influencias siniestras que tomó su nombre del film de Franklin J. Schaffner Los niños 
del Brasil (The boys from Brasil, 1978), una desasosegante película que fabulaba con la posibilidad que el terrible doctor Menguele hubiera huido al país sudamericano y allí ensayara uno de sus experimentos: la clonación de Hitler en unos niños y con ellos el resurgimiento de un nuevo reich.

\section{Las portadas.}

La ya citada formación madrileña Gabinete Caligari debutó en el mercado discográfico con un single compartido con otro de los grupos más representativos del afterpunk español, Parálisis Permanente, en el que cada grupo contribuía con dos canciones. La primera edición, lanzada por la novísima discográfica Tres Cipreses, se agotó en pocos días y la segunda edición llevaba una nueva portada, en este caso un fotograma de la película de culto La parada de los monstruos (Freaks, 1932), un film que recreaba un ambiente inquietante y desasosegante, poblado de personajes deformes y extraños, y cuyo desarrollo culmina con una terrorífica escena final. En este caso la portada del disco y la película elegida resumían y condensaban el espíritu y el universo del rock siniestro y su afición por lo grotesco, lo enfermizo, lo oscuro, el horror en definitiva. Como veremos más adelante no será la única referencia de estos grupos a la película de Tod Browning. El último single de Parálisis Permanente antes de su prematura desaparición provocada por la muerte de su líder Eduardo Benavente en accidente automovilístico lleva en la portada un homenaje a una de las grandes estrellas del cine mundial, Marlene Dietrich, posando en una fotografía de estética expresionista.

Los también citados Hombres $\mathrm{G}$ dedicaron algunas portadas a exhibir su cinefilia, así en su primer LP, de título homónimo al grupo, la ilustración seleccionada es un fotograma de El profesor chiflado (The nutty professor, 1963), en el que aparecen sus protagonistas Jerry Lewis y Stella Stevens. Esta misma actriz también aparecía en la portada del single Venezia. El segundo "long play" del grupo madrileño llevaba el título de La cagaste... Burt Lancaster, y en la portada aparecía un fotograma del actor extraído del clásico de aventuras El halcón y la flecha (The flame and the arrow, 1950). Uno de los mitos del séptimo arte, la actriz Audrey Hepburn, ilustra la carátula del single Tengo una chica. En el año 2002 Hombres G lanzó al mercado un disco recopilatorio en cuya portada aparecen James Cagney y Jean Harlow, en un fotograma perteneciente a uno de los títulos más célebres del género negro, El enemigo público (The public enemy, 1931). A este mismo film, dirigido por William A. Wellman para Warner Bros, pertenece la fotografía que ilustra la portada del disco Música de contrabando, del grupo malagueño Danza Invisible. A otro mito del cine clásico dedicó el grupo de punk madrileño Commando 9mm la carátula de su LP Dios salve a los taraos, en el que aparece un fotograma de Charles Chaplin en su celebérrima película El gran dictador (The great dictator, 1940). Una de las grandes estrellas femeninas de la década de los cincuenta fue Grace Kelly, ganadora de un Oscar en 1954, cuya belleza ilustra un single del grupo madrileño Desperados. 
El grupo madrileño Pistones dedicó una canción titulada Lo que quieras oír a la película El crepúsculo de los dioses (Sunset Boulevard, 1950) en la que el director Billy Wilder narraba el olvido de una antigua estrella de Hollywood de la época del cine mudo. La película estaba protagonizada por Gloria Swanson, cuya fotografía aparece en el single que contiene la canción.

Aunque si hay una estrella cinematográfica que se haya convertido con el paso de los años en un icono representativo del Cine, esa es sin duda Marilyn Monroe, la actriz prematuramente desaparecida, que a los cincuenta años de su muerte su imagen sigue protagonizando campañas publicitarias y que cada año su nombre aparece en la muy citada lista Forbes de "Fallecidos más rentables" (10 millones de dólares facturados en 2012, y 27 millones en 2011). En 1987 el grupo de tecno-pop Olé Olé editaba el disco Los caballeros las prefieren rubias, título tomado de la famosa película de Howard Hawks (Gentlemen prefer blondes, 1953), y su cantante Marta Sánchez aparecía en la portada ataviada y posando al estilo de las fotografías de promoción de Marilyn Monroe en sus años dorados.

\section{Los discos.}

Los motivos para titular a un disco con el nombre de una película pueden ser diversos y aunque puede desvelar una notable cinefilia no ocurre esto en todos los casos. En ocasiones el título de una película refleja el contenido de un disco, lo que denominaríamos "un encuentro afortunado". Así la obra maestra de William Wyler Los mejores años de nuestras vidas (The best years of our lifes, 1946) sirvió para titular un disco recopilatorio del grupo vasco Dinamita pa los pollos, que obviamente recogía "lo mejor" de su trayectoria musical.

En otros casos el nombre del film revela un estilo, como el de la formación madrileña de estética y sonido western La Frontera, que tituló Duelo al sol a su EP de presentación, un nombre que tomó prestado del clásico dirigido por King Vidor (Duel in the sun, 1946), producido por David O. Selznick (Lo que el viento se llevó, Rebeca), e interpretada por Jennifer Jones y Gregory Peck.

El grupo barcelonés de rock clásico y rockabilly Los Rebeldes se inspiraron en el filme generacional de Nicholas Ray (Rebel without a cause, 1955) que encumbró a James Dean para nombrar su LP de 1985 Rebeldes con causa, en el que también encontramos una canción con el mismo título.

Los zaragozanos Niños del Brasil (grupo en el que militó Enrique Bunbury, que luego se haría celebérrimo como líder de Héroes del Silencio) editó cuatro discos de larga duración y al último lo titularon como la escandalosa película del japonés Nagisha Oshima El imperio de los sentidos (Ai no korîda, 1976).

Después de unos años de un éxito extraordinario (probablemente junto a Mecano los grupos españoles más vendedores de la década de los ochenta), y tras una larga 
separación, Hombres G volverían a reunirse en 2004 y sacar al mercado un disco en directo que, siguiendo su trayectoria cinéfila, llevaría por título El año que vivimos peligrosamente, tomado de la película australiana de Peter Weir (The year of living dangerously, 1993).

\section{Los géneros.}

Entre las referencias cinematográficas las formaciones musicales muestran sus preferencias por algunos géneros concretos, y dos sobresalen entre los demás: el cine de suspense-terror y el western. En relación al primero hay que resaltar que un movimiento primerizo como el ya nombrado afterpunk o rock siniestro se hizo patente en Gabinete Caligari, pero también son evidentes las muestras en otro de los grupos señeros de la corriente como fueron Parálisis Permanente. En este caso podemos ver canciones como Tengo un pasajero, inspirada en Alien, el octavo pasajero (Alien, 1979); Unidos, que se centra en unos hermanos siameses, y que se inspira en la ya citada anteriormente La parada de los monstruos; y la canción Un día en Texas, recreación musical de la película de culto de Tobe Hooper La matanza de Texas (The Texas chain saw massacre, 1974). Gabinete Caligari incluyó una versión de esta canción en su disco Que dios reparta suerte como homenaje a los desaparecidos Parálisis Permanante, y años más tarde Intronautas hicieron una nueva versión, en este caso titulada Un día en Waco, localizada en la tristemente célebre localidad tejana donde tuvo lugar el enfrentamiento entre la secta de los Davidianos y la policía norteamericana.

Con toda probabilidad el género cinematográfico más genuino sea el western, o utilizando el lenguaje popular las películas "del oeste" o las películas de "vaqueros". El género creó un imaginario y un universo icónico que dejó su impronta en los demás vehículos de la cultura popular, entre ellos la música en general. Quizá el grupo español de la época más representativo de la corriente western sea La Frontera, como ya se refleja desde su inequívoco nombre. Su primer disco, titulado como el grupo, se abre con la canción La ley de la horca (Tribute a bad man, 1956), film clásico interpretado por James Cagney. Una de las constantes identitarias de la formación madrileña son las continuas referencias al citado universo "west" creado por las películas y que si bien no citan a una película concreta, sí vemos en las canciones la huella simbólica de las mismas: canciones como Vivos o muertos, Rodeo criminal, El fantasma del ahorcado, Pobre tahúr o Judas el Miserable contienen y reflejan el citado universo. Algo parecido sucede con Los Rebeldes, como vemos en su disco en directo de 1986 Preferiblemente vivos, en cuya portada podemos observar la inspiración de los clásicos carteles de "Se busca" (wanted) que colgaban en los "saloons" del salvaje oeste y que ponía precio a la cabeza de los forajidos, y que hemos visto en tantas películas del género. Por su parte Los Enemigos dedicaron una de sus canciones más célebres al gran icono del western, John Wayne. 


\section{Los grupos españoles en el cine.}

En la década de los sesenta era habitual que los grupos de música pop interviniesen en películas para promocionar sus discos y aprovechar el tirón de la fama para llenar las salas. A partir de la década de los setenta y primeros ochenta esta relación se fue diluyendo y los grupos pop y rock fueron sustituidos por formaciones y cantantes dirigidos al público infantil; así Parchís, Enrique y Ana, Chispita y sus gorilas, y tantos otros hoy olvidados fueron protagonistas de filmes que buscaban la explotación de un fenómeno que se encontraba en pleno apogeo. Sin embargo sí existen algunos casos de grupos de los ochenta que participaron en bandas sonoras e incluso algunos músicos intervinieron como actores y actrices de algunos filmes.

En primer lugar hay que destacar el caso de Pedro Almodóvar, activista primigenio de la Movida, y componente junto a Fabio McNamara del estrambótico e iconoclasta dúo Almodóvar y McNamara. En su película de debut, Pepi, Luci, Bom y otras chicas del montón (1980), Almodóvar contó con Alaska para interpretar uno de los personajes principales (Bom), y su grupo, Alaska y los Pegamoides interpretan dos canciones de la banda sonora. En su segunda película, Laberinto de pasiones (1982), el propio Almodóvar y su compañero Fabio McNamara interpretan dos canciones, y en la banda sonora tiene una intervención destacada Bernardo Bonezzi, líder de Zombies.

El director Fernando Colomo filmó en 1978 Qué hace una chica como tú en un sitio como éste, un film que con el paso de los años ha quedado en la memoria colectiva como una de las películas más representativas de la Transición. El grupo madrileño Burning interpretó el tema que daba nombre a la película, además de aparecer en la misma, y la canción se ha convertido en la más célebre de la formación, una de las más veteranas del rock español, todavía en activo.

Como un caso excepcional y extraordinario hay que calificar el caso de Hombres G y su relación con el cine. En la cima de su carrera, con cientos de miles de discos vendidos entre España e Hispanoamérica, y con un referente como el director Manuel Summers, no debe extrañar que el grupo y su entorno quisieran aprovechar la mayúscula popularidad que gozaban para extender su radio de acción hacia las salas de proyecciones y aumentar así celebridad y cuentas corrientes. La primera película que protagonizaron fue Sufre mamón (1987), del mismo título de la canción que les lanzó a la fama, y a la que podemos denominar una película "familiar": en los créditos encontramos a Manuel Summers como director, guionista y productor; a Francisco y Tomás Summers como guionistas; a Curro M. Summers y Guillermo Summers como actores secundarios; y al líder del grupo, David, como protagonista. La película fue un éxito de taquilla (según la web mundocine.net, superó el millón de espectadores y recaudó casi trescientos millones de pesetas) y en consecuencia el "clan" Summers rodó inmediatamente la segunda película a mayor gloria de Hombres G, en este caso Suéltate el pelo (1988). Esta segunda película aunque obtuvo unos números notables en público y dinero no llegó a superar los niveles de éxito de la primera (en torno a 815.000 espectadores y algo más de los 215 millones de pesetas de recaudación, según mundocine.net). 
Como mera operación comercial y en busca de la rentabilidad máxima en menoscabo de la mínima calidad artística cabe calificar el estreno de la película A tope (1984), dirigida por Tito Fernández, que en plena explosión de la Movida madrileña y otros movimientos musicales periféricos intentó aprovechar el creciente éxito de grupos como Alaska y Dinarama, Golpes Bajos, Nacha Pop, Aviador Dro, Video, Loquillo y los Trogloditas, Objetivo Birmania y Gabinete Caligari para arrastrar a sus crecientes seguidores a las taquillas de los cines. El filme no consiguió su objetivo y solo obtuvo unos resultados "mediocres": alrededor de 350.000 espectadores que dejaron una recaudación de unos 70 millones de pesetas.

También hay que reseñar la participación de los músicos españoles en bandas sonoras, como el caso del líder de Los Rebeldes, Carlos Segarra, que en 1990 recibió el encargo de los productores de Las edades de Lulú, película basada en el relato de Almudena Grandes, llevada a la pantalla por Bigas Luna, para musicar el film. El disco resultante contenía trece cortes.

Hay que calificar como sorprendente la inclusión del grupo femenino Las Chinas en la banda sonora de La próxima estación (1982), filme del veterano Antonio Mercero, famoso por haber dirigido dos de las series televisivas más exitosas de la televisión en España, Verano Azul y Farmacia de Guardia. Las Chinas, de efimera trayectoria que incluye un solo single, participaron con la canción Te espío.

El grupo madrileño Los Enemigos fueron los protagonistas absolutos de las bandas sonoras de dos filmes. El primero, Tengo una casa, dirigido por la debutante Mónica Laguna en 1996, demuestra la admiración de la directora por la formación liderada por Josele Santiago, ya que el nombre de la película coincide con un tema del álbum Ferpectamente, el guión gira en torno a un músico seguidor de Los Enemigos, y como hemos apuntado, la banda sonora es exclusiva del grupo, en la que encontramos diecinueve cortes. La segunda película es Se buscan fulmontis, del también novel Álex Calvo-Sotelo, realizada en 1999 y en el que la formación madrileña interpreta quince temas, algunos propios y otros adaptaciones y versiones.

A principios de los años noventa el director vasco Álex de la Iglesia cosecharía un gran éxito con sus dos primeras películas. En su filme de debut, Acción mutante (1993), el grupo Def con Dos pondría música a la canción del mismo título, y para su segundo filme, El día de la Bestia, una de las películas más taquilleras de la década, volvió a contar con Def con Dos para que interpretara la canción que abría la banda sonora y que ponía título a la película.

Podemos afirmar que el músico español con más actividad cinematográfica es Javier Gurruchaga, el líder de la donostiarra Orquesta Mondragón, que debutó como actor en 1982 en la película de Fernando González Bésame tonta (título inspirado en la comedia Bésame tonto, filme de Billy Wilder de 1964, con Dean Martin y Kim Novak), y en la que el grupo donostiarra es responsable de la banda sonora. A partir de entonces son numerosas las apariciones de Javier Gurruchaga en el cine español, e incluso ha conseguido dos nominaciones a los premios Goya al mejor actor secundario: la primera por El rey pasmado, de Imanol Uribe (1991), y la segunda por Tirano 
Banderas, de José Luis García Sánchez (1993). Entre otras películas a destacar de la carrera cinematográfica de Gurruchaga podemos citar: ¿Qué he hecho yo para merecer esto? de Pedro Almodóvar (1984); Si te dicen que caí, de Vicente Aranda (1989); París-Tombuctú, de Luis García Berlanga (1999); y Cuarteto de La Habana, de Fernando Colomo (1999).

La cinefilia del cantante de la Orquesta Mondragón se hace patente en su discografía donde encontramos varias referencias. Así el tema Garras humanas recrea La parada de los monstruos (Freaks, 1932); La viuda alegre toma prestado el título de la obra maestra del cine mudo de Erich von Stroheim (The merry widow, 1925); El diablo dijo no se inspira en Heaven can wait (1943), de Ernst Lubitsch; y El Padrino tiene como claro referente la magna obra de Francis Ford Coppola (The Godfather, 1972). Sin embargo no todas las canciones "cinematográficas" de la Orquesta Mondragón se inspiran en hitos artísticos del cine, así junto a las cuatro cimas citadas encontramos una canción dedicada a otro hito, en este caso comercial: Rambo (1982), de Sylvester Stallone, al que dedican una canción en su álbum de 1987 Ellos las prefieren gordas. A comienzos de 2011 la Orquesta Mondragón editaban nuevo disco con título cinéfilo, El maquinista de la General, un homenaje al clásico de Buster Keaton (The General, 1926).

Otro de los músicos españoles con mayor actividad cinematográfica es Coque Malla, el que fuera líder de Los Ronaldos. Debutó como protagonista en el corto El columpio (1992), junto a la actriz Ariadna Gil, dirigido por Álvaro Fernández Armero. Con este mismo director protagonizó Todo es mentira (1994), junto a Penélope Cruz y Jordi Mollá; y Nada en la nevera (1998), junto a María Esteve. También ha trabajado con el director Francisco Regueiro en Madregilda (1993), que protagonizaron Juan Echanove y José Sacristán; El efecto mariposa (1995) de Fernando Colomo, junto a María Barranco y Rosa María Sardá; y La leyenda de Balthasar el Castrado (1996), de Juan Miñón, junto a Imanol Arias. Sus canciones han formado parte de la banda sonora de varias películas, entre las que citaremos Rec 3 (2012) y Los dos lados de la cama (2005), y es el responsable y compositor de la música de El columpio y Nada en la nevera.

\section{Panorámica por la historia del cine.}

Como ha quedado ya demostrado por lo antes expuesto el cine es un tema recurrente en la discografía de las formaciones españolas cuyas carreras se desarrollaron fundamentalmente en la década de los ochenta. Como colofón a este artículo vamos a hacer un recorrido cronológico por las películas citadas en los discos, si bien omitiremos algunas de las ya nombradas con anterioridad. Así la obra maestra del cine futurista dirigida por Fritz Lang Metrópolis (1927) aparece en el repertorio de Kikí d'Akí, y los cordobeses Pabellón Psiquiátrico dedican una canción al Ángel Azul (Der blaue 
engel, 1930), el film alemán que descubrió a Marlene Dietrich y a su director Josef von Sternberg y que abrió las puertas de Hollywood para ambos.

En los años treinta el director James Whale realizó para la Universal algunas de las películas de terror y ciencia ficción que marcaron el género e influenciaron en muchos directores de entonces y posteriores. Una de aquellos filmes fue El hombre invisible (1933), adaptación de la novela de H.G. Wells que sorprendió a los espectadores por sus efectos especiales, y encontramos canciones con el mismo título en las discografías de los granadinos 091 y Kiko Veneno.

Uno de los primeros éxitos del grupo mallorquín La Granja llevaba por título ¿Por quién doblan las campanas? titulo prestado de la adaptación que Sam Wood hizo en 1943 de la novela homónima de Ernst Hemingway (For whom the bells tolls), cuya trama se desarrolla en la Guerra Civil española y que protagonizaron Gary Cooper e Ingrid Bergman.

La reina de África (The African queen) reunió en 1951 a tres de las personalidades más respetadas de la Historia del Cine: el director John Huston, el actor Humphrey Bogart (ganador del Oscar el mejor actor por esta película) y la actriz Katharine Hepburn. Dos grupos vascos, 21 Japonesas y Duncan Dhu tienen una canción con el mismo título.

La formación asturiana Los Ilegales se inspiran para una de sus canciones en la célebre obra maestra de Luis Buñuel, rodada en su exilio mexicano, El ángel exterminador (1962), un título que coincide con el primer disco editado por Carlos Berlanga en solitario tras su paso por Pegamoides y Dinarama.

En 1969 el director Sidney Pollack retrató la miseria económica y moral de un país en crisis y la lucha por sobrevivir convertida en competición en Danzad, danzad, malditos (They shot horses, don't they). Como vemos el título original y su adaptación al castellano -como ocurre en muchas películas- no guardan ninguna relación, aunque en este caso el título elegido por los distribuidores españoles resultó de una sonoridad tan llamativa que dos grupos de muy distinto estilo se inspiraron en el mismo. Por un lado una de las canciones más recordadas de Glutamato Ye-yé, y por otro el título de un LP de los valencianos Presuntos Implicados llevan el nombre de Danzad, danzad, malditos.

Si ya la publicación de la novela de Anthony Burges La naranja mecánica había supuesto un escándalo de dimensiones mayúsculas a comienzos de la década de los sesenta, cuando el controvertido director Stanley Kubrick la adaptó a la pantalla (A clorkwork orange, 1971) la polémica se reactivó y se multiplicó. El filme supuso un éxito de taquilla y a la vez la imaginería empleada por Kubrick y su tratamiento de la ultraviolencia la convirtieron en una película de culto. Como era lógico un filme sobre la violencia, los jóvenes y las bandas atrajo a los grupos musicales de la época que estamos analizando y así Los Nikis tienen en su discografía el tema La naranja ya no es mecánica, y los futuristas Aviador Dro dedican una canción a Álex y los Drugos. 


\section{Conclusión.}

Podemos afirmar que la generación que irrumpió a finales de los años setenta en España es la primera generación audiovisual, es decir, que si la televisión inicia sus emisiones en nuestro país a finales de la década de los cincuenta y se populariza durante los sesenta, los jóvenes que rondan la veintena cuando llega la Democracia han crecido junto a la televisión y sustituyen así a las generaciones radiofónicas. El cine, contenido habitual de la única televisión de entonces, con filmes de todos los tiempos y géneros se ancla en el imaginario colectivo y emerge como referente. De esta forma se explica que los grupos musicales de los ochenta tengan en sus discografías tantas referencias fílmicas. Y solo así se explica también que entre ellas destaquen preferentemente títulos y estrellas del cine clásico norteamericano, tan común en la televisión de entonces como inexistente en los canales actuales.

En el amplio catálogo encontramos películas de culto, como La parada de los monstruos y La naranja mecánica; hitos del cine mudo como Metrópolis y El ladrón de Bagdad; obras maestras como La reina de África y El Padrino; e iconos del star-system como Marlene Dietrich, Marilyn Monroe y John Wayne.

\section{Bibliografía.}

ALAY PARRA, A. (2011). Rockin'Spain. Madrid: Lunwerg.

GALLEGO DÍAZ, J.L. (1991). Solo se vive una vez. Esplendor y ruina de la movida madrileña. Madrid: Ardora.

MARTÍNEZ SÁNCHEZ, J. (2012): Cervezas, chicas y rockabilly. Historia del rock'n'roll en España. Barcelona: Cuarentena.

ORDOVÁS, J. (2013): Viva el pop. Madrid: Lunwerg.

ORDOVÁS, J. (1987): Historia de la música pop española. Madrid: Alianza Editorial.

\section{El autor}

Dr. Juan Carlos Rodríguez Centeno. Profesor Titular de la Facultad de Comunicación de la Universidad de Sevilla. Profesor Invitado en universidades de Venezuela y Perú. Miembro del equipo de investigación IDECO (Grupo de investigación en comunicación política, ideología y propaganda). Autor de más de una veintena de monografías, artículos, libros, capítulos de libros, etc.Especialista en comunicación empresarial e institucional y cultura de masas. 